\title{
Characteristics of US Health Care Providers Who Counsel Adolescents on Sports and Energy Drink Consumption
}

\author{
Nan Xiang, ${ }^{1,2,3}$ Holly Wethington, ${ }^{3}$ Stephen Onufrak, ${ }^{3}$ and Brook Belay ${ }^{3}$ \\ ${ }^{1}$ University of Michigan Medical School, Ann Arbor, MI 48109, USA \\ ${ }^{2}$ The CDC Experience Applied Epidemiology Fellowship, Scientific Education and Professional Development Program Office, \\ Centers for Disease Control and Prevention, Atlanta, GA 30341, USA \\ ${ }^{3}$ Division of Nutrition, Physical Activity, and Obesity, National Center for Chronic Disease Prevention and Health Promotion, \\ Centers for Disease Control and Prevention, 4770 Buford Hwy NE, MSK-26, Atlanta, GA 30341, USA \\ Correspondence should be addressed to Brook Belay; bbelay@cdc.gov
}

Received 25 October 2013; Revised 3 February 2014; Accepted 4 February 2014; Published 24 March 2014

Academic Editor: Steven E. Lipshultz

Copyright (c) 2014 Nan Xiang et al. This is an open access article distributed under the Creative Commons Attribution License, which permits unrestricted use, distribution, and reproduction in any medium, provided the original work is properly cited.

\begin{abstract}
Objective. To examine the proportion of health care providers who counsel adolescent patients on sports and energy drink (SED) consumption and the association with provider characteristics. Methods. This is a cross-sectional analysis of a survey of providers who see patients $\leq 17$ years old. The proportion providing regular counseling on sports drinks (SDs), energy drinks (EDs), or both was assessed. Chi-square analyses examined differences in counseling based on provider characteristics. Multivariate logistic regression calculated adjusted odds ratios (aOR) for characteristics independently associated with SED counseling. Results. Overall, $34 \%$ of health care providers regularly counseled on both SEDs, with $41 \%$ regularly counseling on SDs and 55\% regularly counseling on EDs. On adjusted modeling regular SED counseling was associated with the female sex (aOR: 1.44 [95\% CI: 1.07-1.93]), high fruit/vegetable intake (aOR: 2.05 [95\% CI: 1.54-2.73]), family/general practitioners (aOR: 0.58 [95\% CI: 0.41-0.82]) and internists (aOR: 0.37 [95\% CI: 0.20-0.70]) versus pediatricians, and group versus individual practices (aOR: 0.59 [95\% CI: 0.42-0.84]). Modeling for SD- and ED-specific counseling found similar associations with provider characteristics. Conclusion. The prevalence of regular SED counseling is low overall and varies. Provider education on the significance of SED counseling and consumption is important.
\end{abstract}

\section{Introduction}

Sugar-sweetened beverages (SSB) are drinks sweetened with various forms of sugars that add calories and include, but are not limited to, soda, fruit ades and fruit drinks, and sports (SD) and energy drinks (ED). On average, SSB intake contributes approximately 300 kilocalories to the daily intake of adolescents 12-19 years old in the United States [1]. Regular consumption of these caloric drinks can increase the risk for obesity [2] and dental caries [3]. In particular, sports and energy drinks (SEDs) are relatively new products that are increasingly marketed to adolescents [4]. Furthermore, purchase and consumption of these drinks by adolescents appear to be common $[1,5,6]$. In 2010, the proportion of high school students who consumed SDs and EDs at least once per day was $16 \%$ and $5 \%$, respectively [7].
SDs contain carbohydrates, minerals, and electrolytes and are often marketed for the purpose of rehydration [8]. However, drinking water alone provides adequate replenishment in most instances other than prolonged vigorous exercise [8]. Both SDs and EDs have been associated with increased dental erosion, due to their acidity [9] and the presence of citric acid [10]. EDs carry additional consequences due to their stimulatory and performance enhancing effects, derived from ingredients such as caffeine, taurine, guarana, and carbohydrate sweeteners $[11,12]$. Unlike caffeinated soda drinks, EDs do not have restrictions on their caffeine concentration nor are they required to have undergone safety testing or placement of appropriate warning labels $[6,13]$. As a result, the caffeine content found in EDs can range from $50 \mathrm{mg}$ to $505 \mathrm{mg}$ per can, which can be as much as that found in 14 12-ounce cans of a typical soda [6]. Consumption 
of EDs may put adolescents at risk of adverse effects of caffeine (elevated heart rate, anxiety, and sleep disturbances) and in one study their consumption was associated with coconsumption of alcohol [6]. A significant proportion of US adolescents regularly consume EDs, ranging from $28 \%$ of 12 to 14 -year-olds to $34 \%$ of 18 - to 24 -year-olds [13]. The problem extends to other countries, as evidenced by one study in Germany that found 23\% of adolescents regularly consuming EDs at $<1$ can per week and 3\% consuming 1 to 7 cans per week [14]. Furthermore, a recent study of US poison center data described the disproportionate exposure of children and young adults to EDs; among cases involving nonalcoholic EDs, over $50 \%$ occurred with children under 6 years of age while $68 \%$ of cases involving alcoholic EDs occurred with young adults under 20 years of age [15].

Health care providers can play an important role in addressing the issue of SED consumption, through counseling and educating adolescent patients and their families on associated health risks. In 2011, the American Academy of Pediatrics (AAP) recommended routine counseling of children and adolescents for SED consumption; the AAP emphasized that water should be the principle source of hydration rather than SD and that due to the potential health risks of caffeine, any consumption of ED by children should be discouraged [8]. While counseling practices related to SEDs have not been well described, numerous studies have examined counseling practices for general SSB or adolescent obesity. One cross-sectional survey found that $38 \%$ of youth participants had discussed SSB with their physicians and $69 \%$ of youths reported that they would change their obesityrelated behaviors if it were recommended by their physicians [16]. In a separate survey study, youths were more likely to report positive perceptions of a health care provider if they discussed one or more "sensitive health topics," which included mood, drugs and alcohol, sexuality, and family issues although body weight was not included [17].

A better understanding is needed on how provider characteristics are associated with counseling practices, specifically with regard to SED consumption. The purpose of this study is to examine the prevalence and frequency of SED counseling among health care providers, where counseling is defined as discussions during which patients are advised to limit consumption of SDs and to avoid any consumption of EDs. Furthermore, this study also examines whether provider characteristics are associated with regular SED counseling.

\section{Methods}

Data was obtained from DocStyles 2011, a web-based panel survey developed by Porter Novelli. The survey is designed to provide insight into health care provider attitudes and counseling behaviors regarding a variety of adult and pediatric health issues and to assess their use of health information resources. In addition, the survey includes questions on the provider's height and weight, as well as other questions describing their demographics, health behaviors, and practice characteristics. The Centers for Disease Control and Prevention Human Subjects Review determined that these analyses were exempt from Human Subjects Review because this is a secondary data analysis using data without identifiers.

2.1. Survey Participants. This study is based on responses from family/general practitioners (FGPs), internists, pediatricians, and nurse practitioners in DocStyles 2011. For the complete DocStyles 2011 survey, physicians and other health care providers were randomly selected from Epocrates' Honor Panel and Epocrates' Allied Health Panel, respectively. Epocrates' Honors Panel is an opt-in panel of over 190,000 medical practitioners, verified by checking each physician against the American Medical Association's (AMA) master file. Participants for this study were randomly selected through a probability sampling method to meet preestablished quotas for each health care provider type $(1,000$ primary care physicians, 250 pediatricians, 250 obstetricians/gynecologists, 250 retail pharmacists, 250 nurse practitioners, and 200 registered dietitians) and for the physician sample to match the AMA master file in terms of age, gender, and region. The total sample is not intended to be representative of the national population of health care providers. Participants were paid an honorarium of $\$ 40-\$ 50$ for completing the survey.

A total of 2,204 health care providers completed the DocStyles 2011 survey. Porter Novelli compared the physician participants, stratified by specialty, to the AMA master file for gender, age, and region of the country and found the average age to be lower compared to national averages. The present study was limited to FGPs $(n=544)$, internists $(n=85)$, pediatricians $(n=239)$, and nurse practitioners $(n=178)$ who regularly see patients $\leq 17$ years old ( $n=1046)$. Only these individuals were eligible to respond to the questions regarding counseling of adolescents on SED consumption, per survey protocol. After exclusion of respondents due to ambiguous or inconsistent responses, the final analytic sample was 1014. The authors were unable to assess the comparability of this analytic sample with the AMA master file because the study was restricted to those who see pediatric patients and are of particular specialties. Comparison data were not available for nurse practitioners.

2.2. Prevalence and Frequency of SED Counseling. The analysis of health care provider counseling of adolescents on SED is based on the three questions listed in Figure 1. Question 1 asks about SED counseling for adolescent patients. Per survey protocol, individuals who responded with "Both drink types" or "Sports drinks only" were asked Question 2, which inquires about frequency of counseling to limit SD consumption. Individuals who responded with "Both drink types" or "Energy drinks only" were asked Question 3, which inquires about frequency of counseling to not drink EDs. The responses were dichotomized in three different ways. Regular SD counseling was defined as those individuals who regularly ("Always" or "Often") counseled on reducing SD consumption with the comparison group being those who counseled less frequently ("Sometimes," "Rarely," or "Never") or did not provide any counseling. Regular ED counseling was defined similarly. Lastly, regular comprehensive SED 


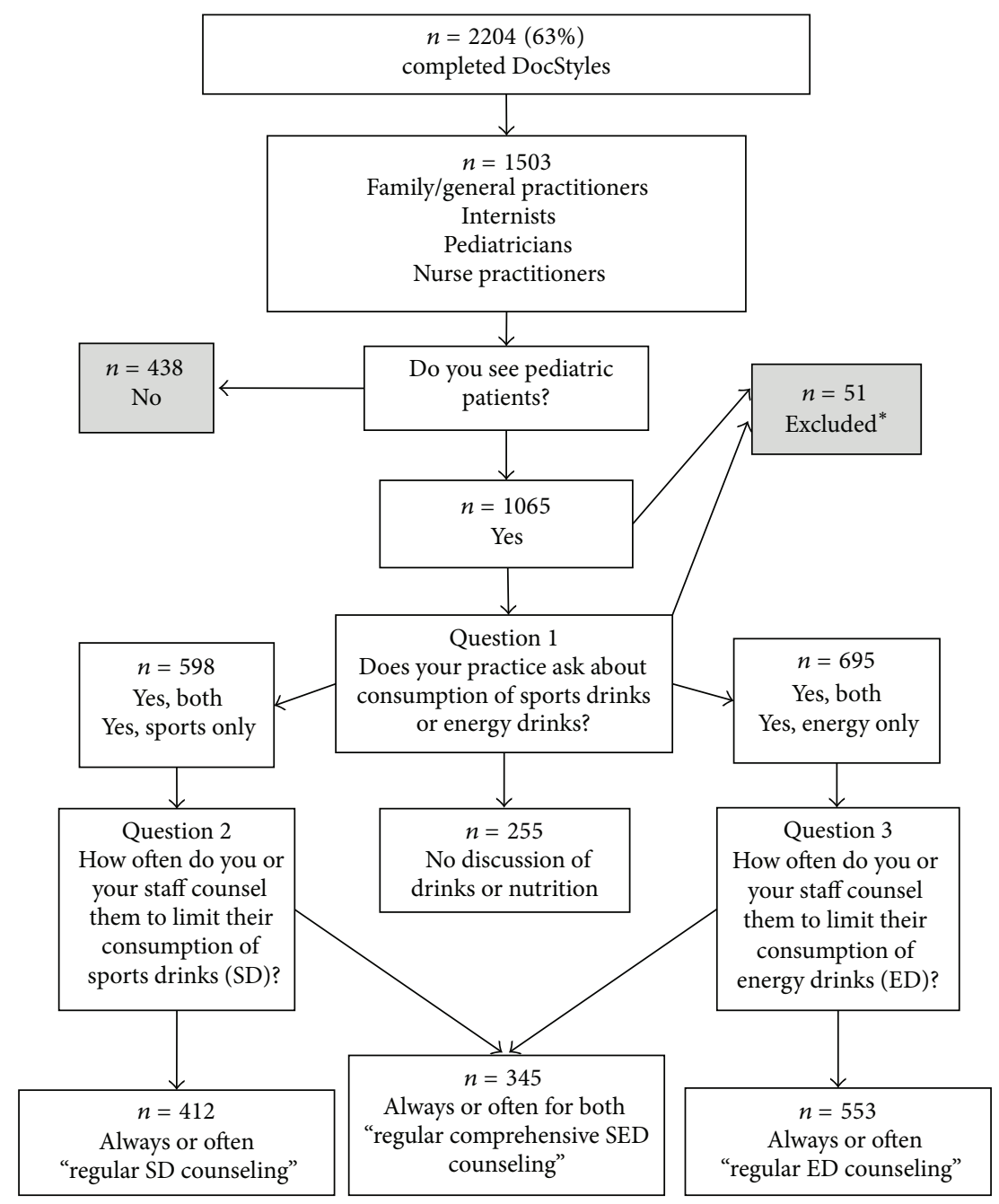

FIGURE 1: Flowchart of DocStyles 2011 survey questions regarding sports and energy drink counseling practices among healthcare providers. This flowchart depicts the questions used in this analysis of the DocStyles 2011 survey and how providers were categorized regarding their counseling patterns around sports (SD) and energy (ED) drinks. This categorization formed the basis of the analyses. Individuals answering "both" or "SD only" to Question 1 were asked Question 2. Those who responded with "both" or "ED only" were asked Question 3. Regular SD counseling was defined as those individuals who regularly ("Always" or "Often") counseled on reducing SD consumption. Regular ED counseling was defined similarly. Comprehensive SED counseling was defined as individuals who counseled on "Both drink types" and counseled regularly for both. $*$ indicates patients excluded from sample due to missing/incomplete demographic information $(n=10)$, responses to Questions 2 or 3 that are contradictory to responses to Question $1(n=9)$, or answering "Not Sure" to Question 1 ( $n=32$ ).

counseling was defined as individuals who counseled on "Both drink types" and counseled regularly for both; the comparison group included everyone who provided counseling less frequently or did not provide counseling. In all three outcomes, individuals who responded with "Not Sure" in Question 1 $(n=32)$ or "Sports drinks/Energy drinks are not typically discussed" in Questions $2(n=2)$ and $3(n=7)$ were excluded due to ambiguity or inconsistency of answers.

2.3. Predictors of SED Counseling. To investigate factors associated with SED counseling, personal and medical practicerelated characteristics of each participant were analyzed. Personal characteristics include age ( $\leq 45$ years versus $>45$ years), sex, race and ethnicity (non-Hispanic white versus all others), weight status determined by body mass index (BMI) calculated from self-reported height and weight ("Nor$\mathrm{mal} /$ underweight" BMI $<25$, "Overweight" $25 \leq \mathrm{BMI}<30$ and "Obese" BMI $\geq 30$ ), number of days per week they ate $\geq 5$ cups of fruit or vegetables henceforth called "high" intake ( $<4$ days versus $\geq 4$ days), and number of days they exercised or kept their heart rate up for 30 minutes $(<5$ days versus $\geq 5$ days). Medical practice-related characteristics included type of specialty, practice setting (individual versus group versus hospital or clinic), affiliation with a teaching hospital, years of practice ( $\leq 10$ years versus $>10$ years), number of total patients per week ( $\leq 100$ versus $>100$ ), and perceived financial status of their patients ("Very Poor to Poor," "Poor to Low Middle," "Low Middle to Middle," "Middle to Upper Middle," and "Upper Middle to Affluent"). 
2.4. Statistical Analysis. The overall prevalence among health care providers giving regular counseling to adolescent patients regarding SDs, EDs, or both was assessed. Chisquare analysis was used to examine differences in the prevalence of regular SED counseling among participants with different personal and medical practice-related characteristics. Multivariate logistic regression was conducted to determine characteristics independently associated with the three outcomes of interest: regular SD counseling, regular ED counseling, and regular comprehensive SED counseling. The significance level was $P<0.05$ and the selection criterion for bivariate inclusion in the multivariate model was $P<0.20$ [13]. All analyses were conducted using SAS 9.2 statistical software (SAS Institute Inc.).

\section{Results}

Approximately three-fourths of providers were non-Hispanic whites, with a relatively even distribution in sex and age (Table 1). Thirty-seven percent of providers were overweight and $15 \%$ were obese, 55\% reported high fruit and vegetable intake (at least 5 cups per day, $\geq 4$ days per week), and $29 \%$ engaged in 30 minutes or more of physical activity $\geq 5$ days per week. Nearly $60 \%$ had been practicing for greater than ten years and approximately half (46\%) had teaching privileges. The majority $(66 \%)$ of the participants worked in a group practice, $57 \%$ had five or less physicians in their practice, and $58 \%$ saw $\leq 100$ patients per week.

\subsection{Regular Sports Drink and Energy Drink Counseling.} Overall, $41 \%$ of participants provided regular (always or often) SD counseling compared to $55 \%$ for ED counseling (Table 1). Approximately $42 \%$ of individuals never counseled about SDs and 32\% about EDs. Female providers exhibited a higher prevalence of regular counseling, at $47 \%$ and $61 \%$ for SDs and EDs, respectively, compared to $35 \%$ and $49 \%$ by male providers. Pediatricians had the highest prevalence of regular SD counseling at 55\% compared to internists who had the lowest prevalence at $30 \%$. For regular ED counseling, nurse practitioners had the highest prevalence at $65 \%$ compared to internists at $43 \%$.

Multivariate modeling found that regular SD counseling was independently associated with being female (adjusted odds ratio (aOR): 1.41 [95\% confidence interval (95\% CI): 1.07-1.88]), high fruit and vegetable intake (aOR: 1.63 [95\% CI: 1.24-2.13]), type of provider, and group practices compared to individual practices (aOR: 0.66 [95\% CI: 0.470.93]) (Table 2). In the model, compared to pediatricians as the reference group, FGPs (aOR: 0.46 [95\% CI: 0.33-0.64]), internists (aOR: 0.33 [95\% CI: 0.18-0.59]), and nurse practitioners (aOR: 0.55 [95\% CI: 0.36-0.83]) all had significantly lower odds of providing regular SD counseling.

Similar to SD counseling, multivariate modeling found that regular ED counseling was independently associated with being female (aOR: 1.40 [95\% CI: 1.06-1.84]), high fruit and vegetable intake (aOR: 1.68 [95\% CI: 1.30-2.16]), internists as compared to pediatricians (aOR: 0.56 [95\% CI: $0.33-0.96]$ ), and group practices compared to individual practices (aOR: 0.68 [95\% CI: 0.48-0.95]) (Table 2). Other provider types did not significantly differ from pediatricians in the adjusted model.

With regard to either regular SD counseling or ED counseling, no statistically significant differences were found with regard to race/ethnicity, BMI, years of practice, teaching privileges, number of physicians per practice, number of patients seen per week, or the provider's perception of their patient population's socioeconomic level (Table 2).

3.2. Regular Comprehensive SED Counseling. The third outcome of this study is regular comprehensive SED counseling, which had an overall prevalence of $34 \%$ (Table 1 ). The prevalence of counseling was higher in female providers at $41 \%$ compared to male providers at $28 \%$. Among the types of health care providers, pediatricians and nurse practitioners had the highest prevalence of comprehensive SED counseling at $43 \%$ and $42 \%$, respectively, compared to FGPs at $29 \%$ and internists at $23 \%$. Multivariate modeling found regular comprehensive SED counseling to be independently associated with being female (aOR: 1.44 [95\% CI: 1.07-1.93]), high fruit and vegetable intake (aOR: 2.05 [95\% CI: 1.54-2.73]), FGPs (aOR: 0.58 [95\% CI: 0.41-0.82]) and internists (aOR: 0.37 [95\% CI: $0.20-0.70])$ compared to pediatricians, and group practices (aOR: 0.59 [95\% CI: 0.42-0.84]) compared to individuals practices (Table 2 ). No other provider characteristics were found to be associated.

\section{Discussion}

We found that one-third of health care providers in this study reported comprehensive SED counseling, indicating that the majority of health care providers who see adolescent patients were not providing both SD and ED counseling regularly. Stratification by provider type found that pediatricians had the highest prevalence of regular comprehensive SED counseling (43\%). This is lower than the SSB counseling rate of $65 \%$ reported by pediatricians in the 2006 AAP Periodic Survey of Fellows [18]. However, differences may be attributed to the topic counseled upon (SED versus SSB) and how "frequent counseling" was defined by each study. In addition to pediatricians, nurse practitioners also exhibited high counseling rates; these two provider types comprised the two highest prevalences of SD only, ED only, and comprehensive SED counseling. Comparatively, FGPs and internists had significantly lower prevalences of SED counseling.

The finding that pediatricians typically exhibited the highest rates of SED counseling is congruent with previous studies, which have also have found that pediatricians more frequently assessed weight status and provided behavioral counseling than family/general practitioners $[19,20]$. Nurse practitioners were more likely to provide SED counseling than FGPs and internists in this study. One possible reason for this difference may be that, in this study, FGPs and internists tend to see fewer pediatric patients per week (FGPs/internists: 23.3 pediatric patient versus nurse practitioners: 29.4 versus pediatricians: 105.0) and may have less experience counseling 


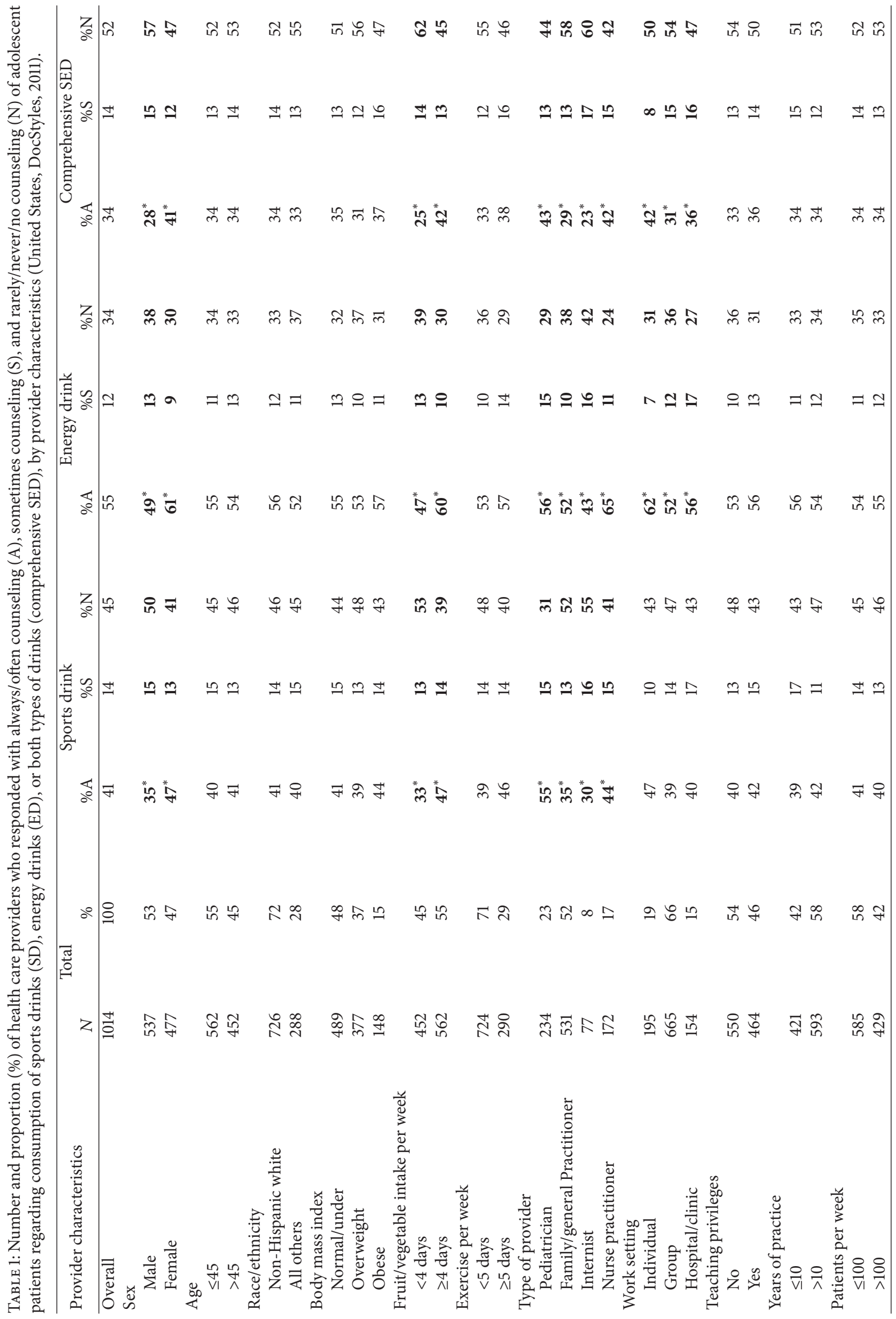




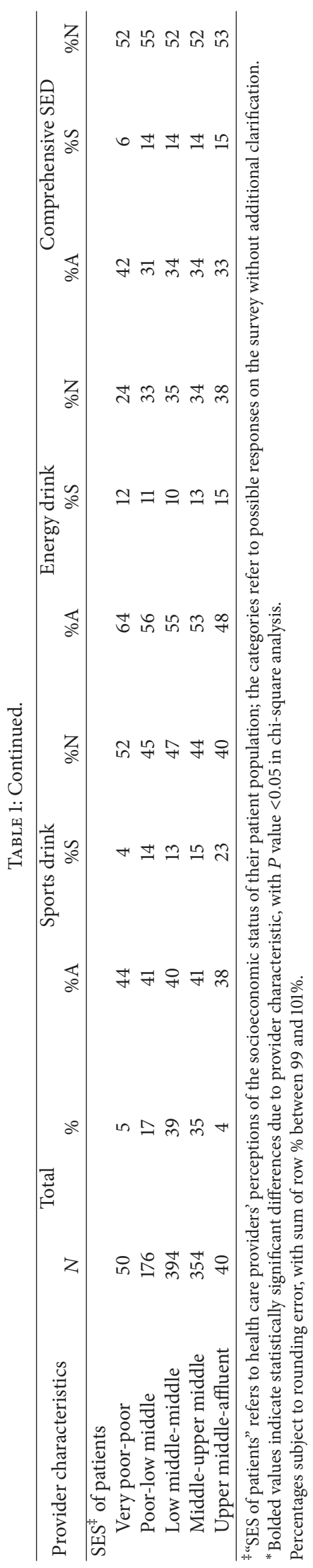




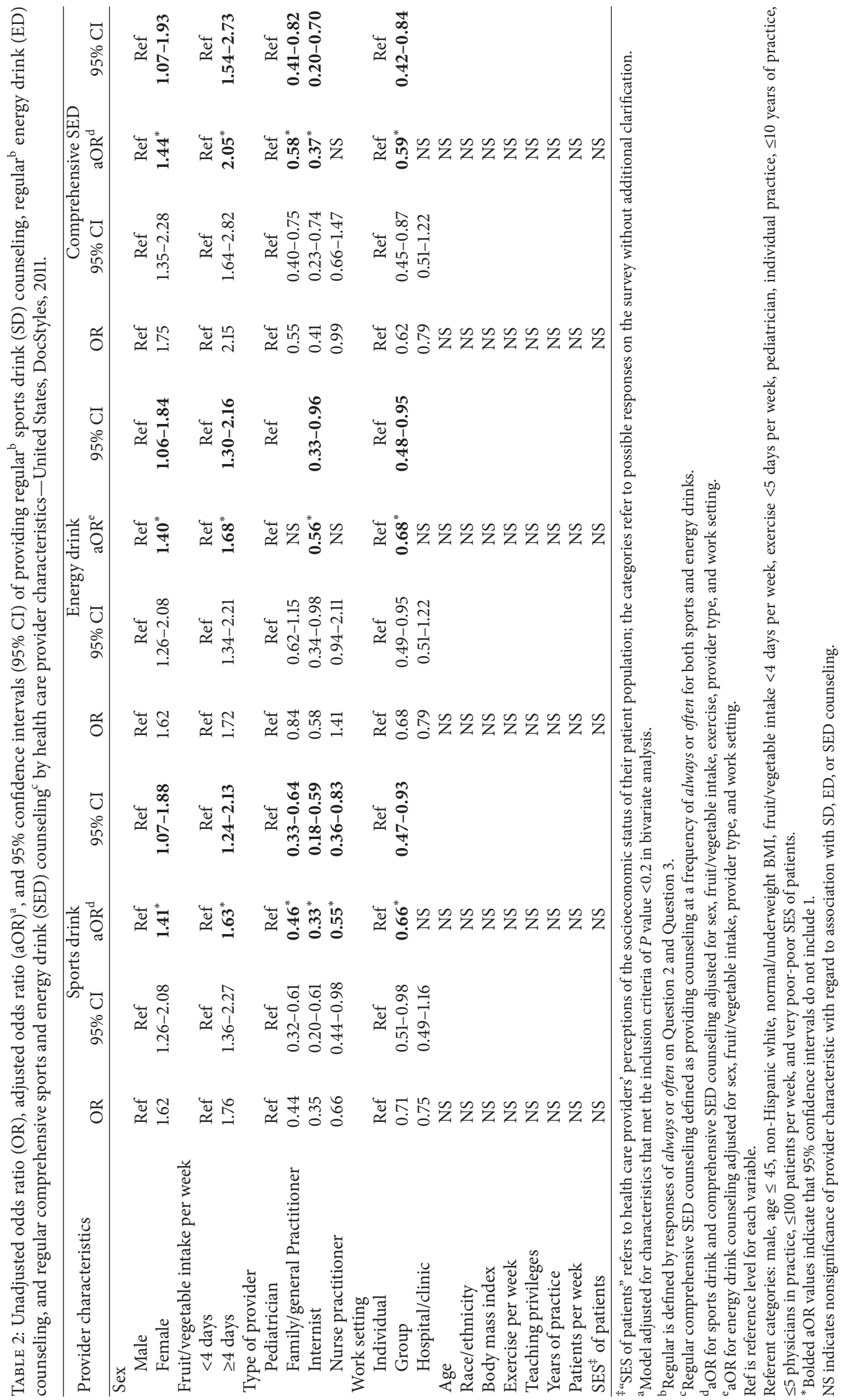


adolescents. Another reason, though not investigated in this study, is the amount of time nurse practitioners have to spend with each patient and the complexity of cases they manage. Regardless of the cause of the disparities, FGPs and internists likely play significant roles in the health maintenance of adolescent patients. Gaps in counseling practices can be addressed through changes in the training and continuing education of providers. Currently, the differences in provider access and familiarity of AAP counseling recommendations are unknown.

This study also found that the prevalence of regular SD counseling and of regular ED counseling significantly differed overall, at $41 \%$ and $55 \%$, respectively. Specifically, this difference varied by provider type, with internists exhibiting the greatest discrepancy (21\% higher for ED counseling) in comparison to pediatricians (1\% higher for ED counseling). These findings suggest that there are provider-specific differences in how the health risks of these two drink types are perceived and managed.

We found that being female, having high fruit and vegetable intake, being a pediatrician, and operating in an individual practice compared to a group practice were associated with greater odds of providing regular comprehensive SED counseling. Regular SD counseling and regular ED counseling were associated with the same provider characteristics. Some variables identified in previous studies [21, 22] to be related to physician counseling behaviors such as race/ethnicity and BMI were not found to be associated in this study. Compared to Bleich et al., this study contained a similar proportion of overweight/obese providers at $52 \%$, with the difference being a focus on adolescent patients with whom providers may be more motivated to counsel on obesityrelated topics.

Given the relatively low rates of regular SED counseling, increasing provider awareness of the health effects of SED is the first step in addressing this issue. Secondly, studies have found that various factors affect how inclined and confident a clinician is in terms of providing health behavior counseling $[23,24]$. One study found that physicians with normal BMIs were more likely to discuss weight loss and were more confident in offering diet and exercise suggestions [21]. Another study found that clinicians who personally struggle with making healthy choices, such as avoiding calorically dense foods and beverages, may find it more difficult to counsel patients on that topic [23]. Our data supports that hypothesis, with providers of high fruit and vegetable intake having higher prevalence of SED counseling. Encouraging health care providers to lead healthy lives may contribute to higher prevalences of SED counseling. Ideally, providers can be role models for healthy behavior and create a supportive environment in their clinic or hospital setting and in the community-at-large for patients and families [25].

Other studies have found that another barrier to offering effective health counseling is a lack of training or confidence in behavioral counseling [23]. Techniques such as motivational counseling [26, 27], the 5 A's heuristic [28], and multistage models [29] provide frameworks from which effective changes can be encouraged. Investigators have developed and examined curricula for medical students and residents
[30-32], comprised of lectures and opportunities to practice counseling techniques that resulted in subjective increases in confidence in providing health behavior counseling. Lastly, physicians can also learn from effective community-based interventions and adapt them for their patients. For example, physicians can consider explaining caloric information to adolescent patients in terms of physical activity equivalents, a promising intervention used for youth in Baltimore [33].

This study has a number of limitations. First, the sample was drawn from an opt-in database, which is subject to selection bias and may not be representative of health care providers nationally. Second, the responses regarding counseling behaviors are subjective and susceptible to recall bias; thus, reported prevalence may not reflect actual practices. Third, there is limited evidence for or against the assignment of the "Sometimes" response to the "NOT regular counseling" group; however, the data was also analyzed with "Sometimes" assigned to the "regular counseling" group and the results were not significantly different. Finally, the survey did not assess barriers that prevented SD and/or ED counseling from being offered.

With the prevalence of regular comprehensive SED counseling at $34 \%$, increased efforts must be applied to educate adolescent patients and not overlook the associated health risks of high consumption of these beverages. Additional research is required to assess physician opinions and barriers that stand in the way of providing regular counseling. Research and training can help teach health care providers of various disciplines pertinent information and effective counseling techniques for patients and parents.

\section{Abbreviations \\ SSB: Sugar-sweetened beverages \\ SD: $\quad$ Sports drinks \\ ED: Energy drinks \\ SED: Sports and energy drinks \\ AAP: American Academy of Pediatrics \\ FGP: Family or general practitioner \\ AMA: American Medical Association \\ BMI: Body mass index \\ aOR: Adjusted odds ratio \\ OR: Odds ratio.}

\section{Disclosure}

The authors have no financial relationships relevant to this paper to disclose.

\section{Disclaimer}

The findings and conclusions in this report are those of the authors and do not necessarily reflect the official position of the Centers for Disease Control and Prevention.

\section{Conflict of Interests}

The authors have no conflict of interests to disclose. 


\section{Authors' Contribution}

Nan Xiang carried out the data analysis, interpreted the results, drafted and revised the initial paper, and composed the final paper as submitted. Holly Wethington was involved in interpretation of the data analysis, providing pertinent references, revision of the paper, and approval of the final paper as submitted. Stephen Onufrak was involved in interpretation of the data analysis, providing pertinent references, revision of the paper, and approval of the final paper as submitted. Brook Belay conceptualized and designed the study questions, facilitated the survey deployment, interpreted the results of data analysis, reviewed and revised the paper, and approved the final paper as submitted.

\section{Acknowledgment}

This work was made possible through The CDC Experience Applied Epidemiology Fellowship, a public/private partnership supported by a grant to the CDC Foundation from External Medical Affairs, Pfizer Inc.

\section{References}

[1] Y. C. Wang, S. N. Bleich, and S. L. Gortmaker, "Increasing caloric contribution from sugar-sweetened beverages and $100 \%$ fruit juices among US children and adolescents, 1988-2004," Pediatrics, vol. 121, no. 6, pp. e1604-e1614, 2008.

[2] V. S. Malik, M. B. Schulze, and F. B. Hu, "Intake of sugarsweetened beverages and weight gain: a systematic review," American Journal of Clinical Nutrition, vol. 84, no. 2, pp. 274288, 2006.

[3] T. A. Marshall, S. M. Levy, B. Broffitt et al., "Dental caries and beverage consumption in young children," Pediatrics, vol. 112, no. 3, part 1, pp. e184-e191, 2003.

[4] Sugary Drink F.A.C.T.S.: Evaluating Sugary Drink Nutrition and Marketing to Youth, R.C.f.F.P. Obesity, Editor, 2011.

[5] R. F. Wescott, "Measuring the purchases of soft drinks by students in US schools," An analysis for the American Beverage Association, 2005.

[6] C. J. Reissig, E. C. Strain, and R. R. Griffiths, "Caffeinated energy drinks-a growing problem," Drug and Alcohol Dependence, vol. 99, no. 1-3, pp. 1-10, 2009.

[7] Centers for Disease Control and Prevention (CDC), "Beverage consumption among high school students-United States, 2010," Morbidity and mortality weekly report, vol. 60, no. 23, pp. 778-780, 2011.

[8] Committee on Nutrition and the Council on Sports Medicine and Fitness, "Sports drinks and energy drinks for children and adolescents: are they appropriate?" Pediatrics, vol. 127, no. 6, pp. 1182-1189, 2011.

[9] L. Shaw and A. J. Smith, "Dental erosion-the problem and some practical solutions," British Dental Journal, vol. 186, no. 3 , pp. 115-118, 1999.

[10] V. K. Järvinen, I. I. Rytömaa, and O. P. Heinonen, "Risk factors in dental erosion," Journal of Dental Research, vol. 70, no. 6, pp. 942-947, 1991.

[11] J. P. Higgins, T. D. Tuttle, and C. L. Higgins, "Energy beverages: content and safety," Mayo Clinic Proceedings, vol. 85, no. 11, pp. 1033-1041, 2010.
[12] E. Cinteza, "Update in pediatrics: to take or not to take soft drinks, sports or energy drinks?" Maedica, vol. 6, no. 2, pp. 157158, 2011.

[13] S. M. Seifert, J. L. Schaechter, E. R. Hershorin, and S. E. Lipshultz, "Health effects of energy drinks on children, adolescents, and young adults," Pediatrics, vol. 127, no. 3, pp. 511-528, 2011.

[14] B. Viell, L. Gräbner, G. Früchel, and P. Boczek, "New caffeinated beverages. A pilot survey of familiarity and consumption by adolescents in North-Rhine Westphalia and Berlin and considerations of consumer protection," Zeitschrift für Ernährungswissenschaft, vol. 35, no. 4, pp. 378-386, 1996.

[15] S. M. Seifert, S. A. Seifert, J. L. Schaechter et al., "An analysis of energy-drink toxicity in the National Poison Data System," Clinical Toxicology, vol. 51, no. 7, pp. 566-574, 2013.

[16] E. M. Taveras, A. M. Sobol, C. Hannon, D. Finkelstein, J. Wiecha, and S. L. Gortmaker, "Youths' perceptions of overweight-related prevention counseling at a primary care visit," Obesity, vol. 15, no. 4, pp. 831-836, 2007.

[17] J. D. Brown and L. S. Wissow, "Discussion of sensitive health topics with youth during primary care visits: relationship to youth perceptions of care," Journal of Adolescent Health, vol. 44, no. 1, pp. 48-54, 2009.

[18] J. D. Klein, T. S. Sesselberg, and M. S. Johnson, "Adoption of body mass index guidelines for screening and counseling in pediatric practice," Pediatrics, vol. 125, no. 2, pp. 265-272, 2010.

[19] T. T. Huang, L. A. Borowski, B. Liu et al., "Pediatricians' and family physicians' weight-related care of children in the U.S.," American Journal of Preventive Medicine, vol. 41, no. 1, pp. 2432, 2011.

[20] H. R. Wethington, B. Sherry, and B. Polhamus, "Physician practices related to use of BMI-for-age and counseling for childhood obesity prevention: a cross-sectional study," BMC Family Practice, vol. 12, article 80, 2011.

[21] S. N. Bleich, W. L. Bennett, K. A. Gudzune, and L. A. Cooper, "Impact of physician BMI on obesity care and beliefs," Obesity, vol. 20, no. 5, pp. 999-1005, 2012.

[22] K. T. Rattay, J. E. Fulton, and D. A. Galuska, "Weight counseling patterns of U.S. pediatricians," Obesity Research, vol. 12, no. 1, pp. 161-169, 2004.

[23] K. S. Vickers, K. J. Kircher, M. D. Smith, L. R. Petersen, and N. H. Rasmussen, "Health behavior counseling in primary care: provider-reported rate and confidence," Family Medicine, vol. 39, no. 10, pp. 730-735, 2007.

[24] E. Frank, E. H. Wright, M. K. Serdula, L. K. Elon, and G. Baldwin, "Personal and professional nutrition-related practices of US female physicians," American Journal of Clinical Nutrition, vol. 75, no. 2, pp. 326-332, 2002.

[25] S. A. Kim, K. A. Grimm, A. L. May, D. M. Harris, J. Kimmons, and J. L. Foltz, "Strategies for pediatric practitioners to increase fruit and vegetable consumption in children," Pediatric Clinics of North America, vol. 58, no. 6, pp. 1439-1453, 2011.

[26] R. P. Schwartz, R. Hamre, W. H. Dietz et al., "Office-based motivational interviewing to prevent childhood obesity: a feasibility study," Archives of Pediatrics and Adolescent Medicine, vol. 161, no. 5, pp. 495-501, 2007.

[27] M. Suarez and S. Mullins, "Motivational interviewing and pediatric health behavior interventions," Journal of Developmental and Behavioral Pediatrics, vol. 29, no. 5, pp. 417-428, 2008.

[28] S. A. Flocke, A. Clark, K. Schlessman, and G. Pomiecko, "Exercise, diet, and weight loss advice in the family medicine outpatient setting," Family Medicine, vol. 37, no. 6, pp. 415-421, 2005. 
[29] S. Lippke and J. P. Ziegelmann, "Understanding and modeling health behavior: the multi-stage model of health behavior change," Journal of Health Psychology, vol. 11, no. 1, pp. 37-50, 2006.

[30] K. Bell and B. A. Cole, "Improving medical students' success in promoting health behavior change: a curriculum evaluation," Journal of General Internal Medicine, vol. 23, no. 9, pp. 15031506, 2008.

[31] E. M. Moser and A. Stagnaro-Green, "Teaching behavior change concepts and skills during the third-year medicine clerkship," Academic Medicine, vol. 84, no. 7, pp. 851-858, 2009.

[32] K. Sibille, A. Greene, and J. P. Bush, "Preparing physicians for the 21 century: targeting communication skills and the promotion of health behavior change," Annals of Behavioral Science and Medical Education, vol. 16, no. 1, pp. 7-13, 2010.

[33] S. N. Bleich, B. J. Herring, D. D. Flagg, and T. L. GaryWebb, "Reduction in purchases of sugar-sweetened beverages among low-income black adolescents after exposure to caloric information," American Journal of Public Health, vol. 102, no. 2, pp. 329-335, 2012. 


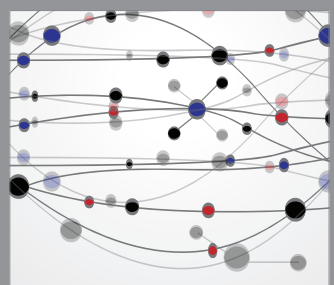

The Scientific World Journal
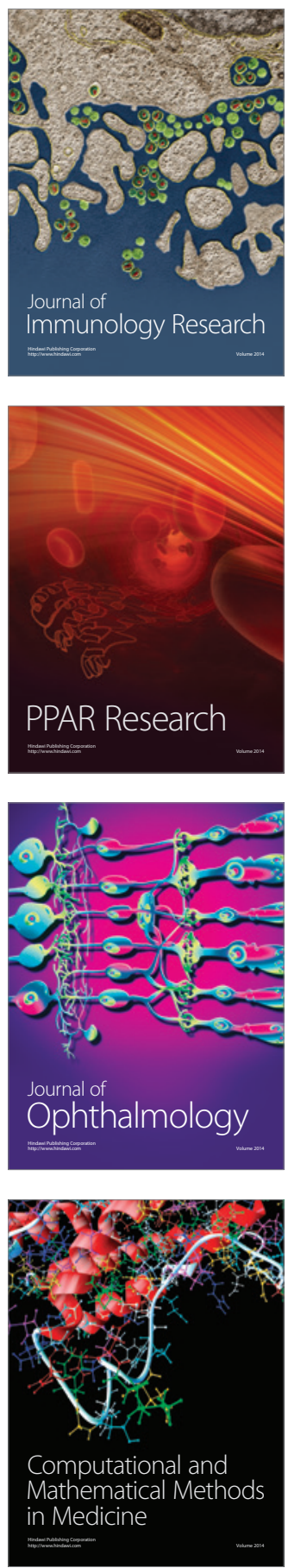

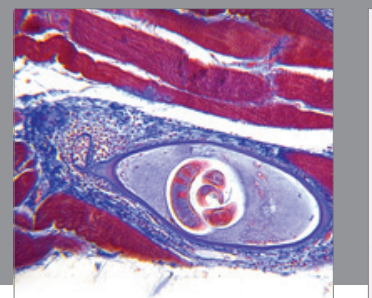

Gastroenterology

Research and Practice
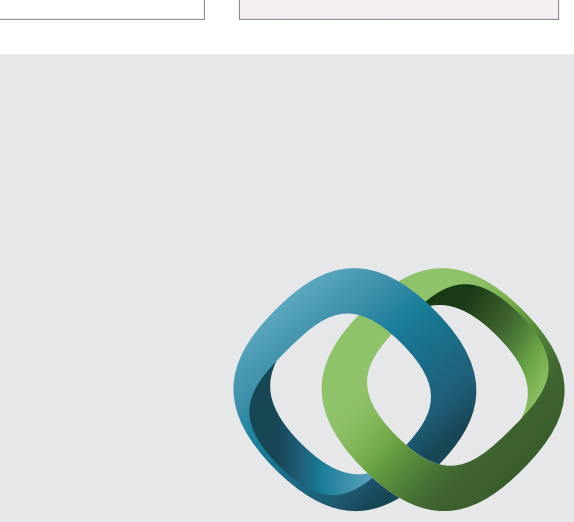

\section{Hindawi}

Submit your manuscripts at

http://www.hindawi.com
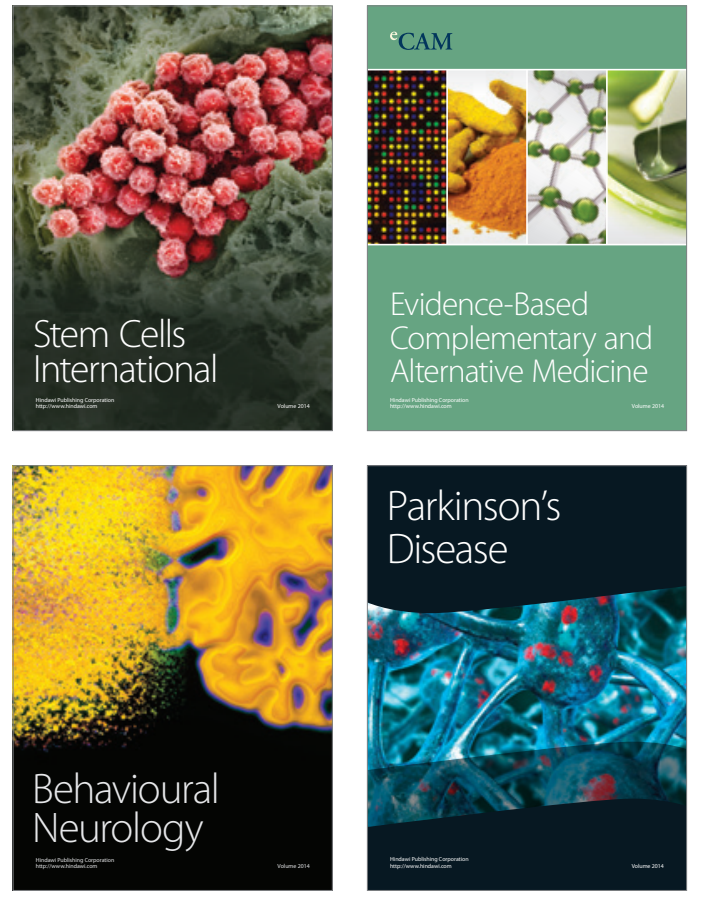
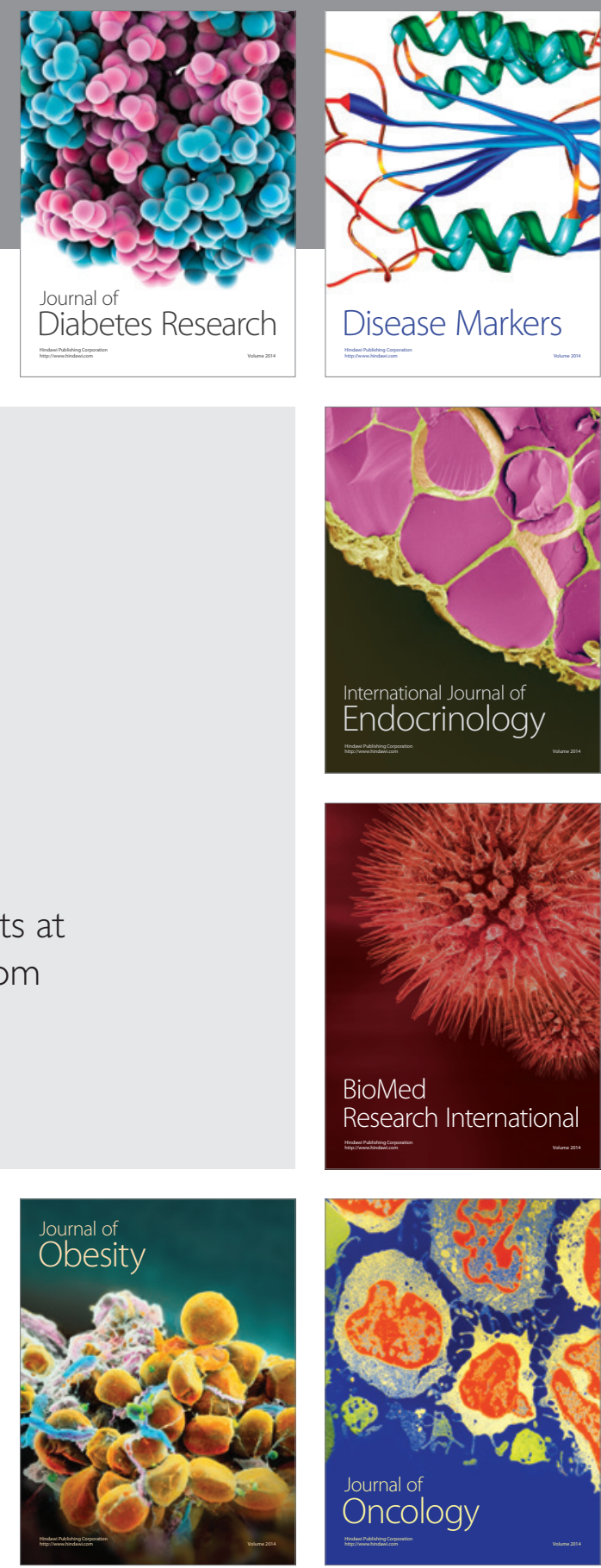

Disease Markers
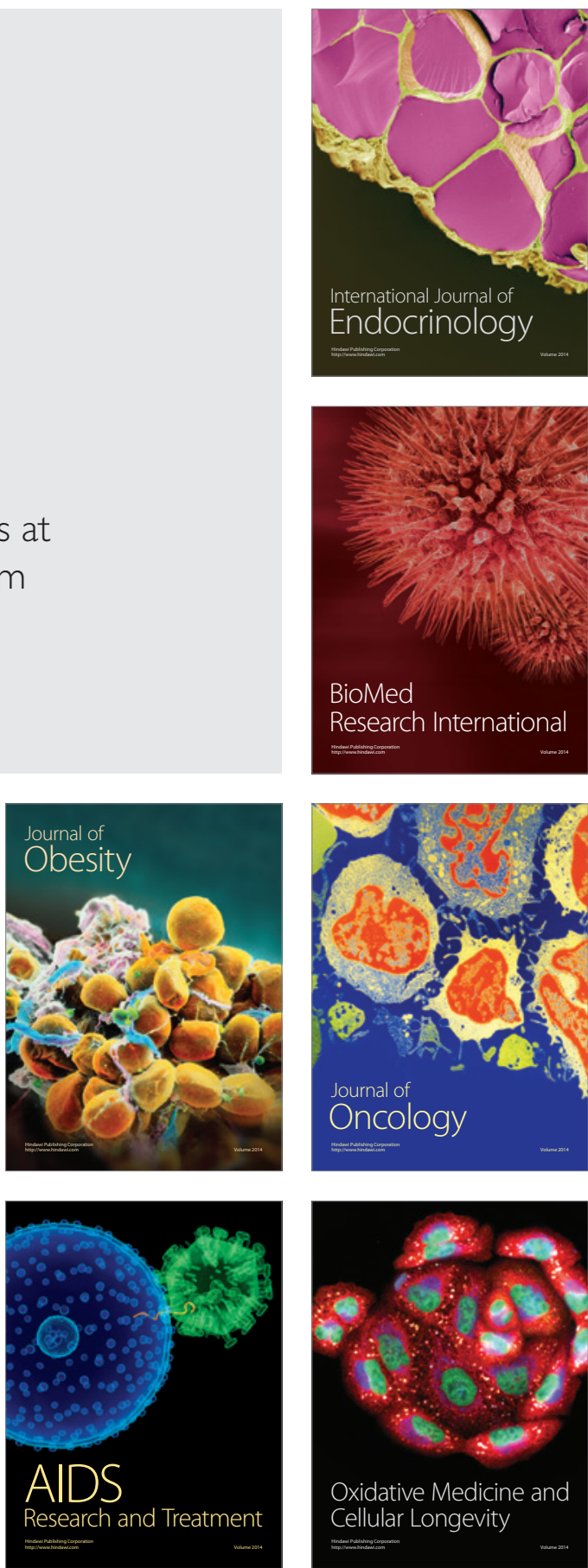